\title{
Plasma LDL-Cholesterol Level at Admission is Independently Associated with Infarct Size in Patients with ST-Segment Elevation Myocardial Infarction Treated with Primary Percutaneous Coronary Intervention
}

\author{
Mathijs C. Bodde - Maaike P. J. Hermans - Ron Wolterbeek • Christa M. Cobbaert • \\ Arnoud van der Laarse $\cdot$ Martin J. Schalij · J. Wouter Jukema
}

Received: October 17, 2018 / Published online: February 13, 2019

(C) The Author(s) 2019

\section{ABSTRACT}

Introduction: Hypercholesterolemia is a wellknown risk factor for developing atherosclerosis and subsequently for the risk of a myocardial infarction (MI). Moreover, it might also be related to the extent of damaged myocardium in the event of a MI. The aim of this study was to evaluate the association of baseline low density lipoprotein-cholesterol (LDL-c) level with infarct size in patients with ST-segment elevation myocardial infarction (STEMI) after

Enhanced digital features To view enhanced digital features for this article go to https://doi.org/10.6084/ m9.figshare.7571069.

Electronic supplementary material The online version of this article (https://doi.org/10.1007/s40119019-0126-5) contains supplementary material, which is available to authorized users.

M. C. Bodde $(\varangle) \cdot$ M. P. J. Hermans .

A. van der Laarse $\cdot$ M. J. Schalij · J. W. Jukema Department of Cardiology, Leiden University Medical Center, Leiden, The Netherlands e-mail: m.c.bodde@lumc.nl

\section{R. Wolterbeek}

Department of Medical Statistics and Bioinformatics, Leiden University Medical Center, Leiden, The Netherlands

C. M. Cobbaert - A. van der Laarse

Department of Clinical Chemistry \& Laboratory

Medicine, Leiden University Medical Center,

Leiden, The Netherlands primary percutaneously coronary intervention (pPCI).

Methods: Baseline blood samples were obtained from all patients admitted between 2004 and 2014 with STEMI who underwent pPCI. Patients were excluded in case of out of hospital cardiac arrest, treatment delay of at least $10 \mathrm{~h}$ or no complete reperfusion after pPCI in the culprit vessel. Peak creatine kinase (CK) level was used for infarct size estimation, defined as the maximal value during admission. Results: A total of 2248 patients were included in this study (mean age $61.8 \pm 12.2$ years; $25.0 \%$ female). Mean LDL-c level was $3.6 \pm 1.1 \mathrm{mmol} / \mathrm{L}$ and median peak CK level was $1275 \mathrm{U} / \mathrm{L}$ (IQR 564-2590 U/L). Baseline LDL-c level $[\beta=0.041$; (95\% CI 0.019-0.062); $p<0.001]$ was independently associated with peak CK level. Furthermore, left anterior descending artery as culprit vessel, initial TIMI 0-1 flow in the culprit vessel, male gender, and treatment delay were also correlated with high peak CK level $(p<0.05)$. Prior aspirin therapy was associated with lower peak $\mathrm{CK}$ level $[\beta=-0.073 \quad(95 \% \quad \mathrm{CI}-0.146$ to 0.000$)$, $p=0.050]$.

Conclusion: This study demonstrates that besides the more established predictors of infarct size, elevated LDL-c is associated with augmented infarct size in patients with STEMI treated with pPCI. 
Keywords: LDL-cholesterol; Myocardial infarct size; Primary percutaneously coronary intervention; ST-segment elevation myocardial infarction

\section{INTRODUCTION}

In patients with ST-segment elevation myocardial infarction (STEMI), primary percutaneously coronary intervention (pPCI) is an effective strategy to reduce the size of myocardial infarction (MI) [1]. Other well-established determinants of infarct size are long duration of ischemia, left anterior descending (LAD) artery as culprit lesion, and low pre- and post-procedural thrombolysis in myocardial infarction (TIMI) flow [2]. Identifying more determinants that influence infarct size could further aid in reducing infarct size. Hypercholesterolemia is a well-known risk factor for developing atherosclerosis $[3,4]$ and subsequently for the risk of a MI [5-8]. Moreover, it might also be related to the extent of damaged myocardium in the event of a MI [9-12].

An important part of a patient's treatment and prognosis is determined by the myocardial infarct size.

Myocardial infarct size can be measured with established biomarkers such as creatine kinase (CK) and cardiac troponin T (cTnT). Peak CK levels are as accurate as cTnT in estimating myocardial infarct size in STEMI patients who underwent successful pPCI [13], and is an established non-invasive measure of infarct size and severity [14-16]. Furthermore, peak CK levels are strongly associated with clinical outcome [14-16]. Myocardial reperfusion injury (MRI) is the term for further injury to the ischemic myocardium which occurs after restoration of blood flow which reduces the beneficial effect of myocardial reperfusion [17]. It is suggested that approximately $30 \%$ of myocardial infarct size is determined by MRI [17] and although the pathophysiological mechanisms of MRI are not fully elucidated [17], this might be preventable. Studies in animal models have demonstrated that hypercholesterolemia may aggravate MRI $[9,10]$, e.g., by the no-flow phenomenon [11] or by increased myocardial oxidative stress $[10,12]$ and inflammation [12].

A relation between hypercholesterolemia and infarct size in clinical studies could have important implications and may lead to more advanced therapies like antioxidant therapy and novel lipid-lowering therapy.

So, we hypothesize that high LDL-cholesterol (LDL-c) levels during admission are associated with infarct size in patients with STEMI. Therefore, the aim of this study was to evaluate the association between baseline LDL-c levels and enzymatic infarct size using peak $\mathrm{CK}$ in patients with STEMI after pPCI.

\section{METHODS}

\section{Study Population}

Consecutive patients admitted with STEMI between February 2004 and January 2014 at the Leiden University Medical Center (LUMC) were included in this retrospective study. All patients were treated with pPCI according to the current guidelines-based institutional MISSION! protocol [18-20]. STEMI was defined as typical electrocardiographic (ECG) changes (ST-segment elevation at least $0.2 \mathrm{mV}$ in at least two contiguous leads in $V_{1}$ through $V_{3}$, at least $0.1 \mathrm{mV}$ in other leads, or presumed new left bundle branch block) and a typical rise and fall of cardiac biomarkers accompanied with chest pain for at least $30 \mathrm{~min}$ [21]. Predefined exclusion criteria were (1) patients presenting with an out of hospital cardiac arrest (OHCA); (2) time of ischemia of $10 \mathrm{~h}$ or more; (3) no complete reperfusion after pPCI in the culprit vessel, defined as TIMI 3 post-procedural flow in the culprit vessel; (4) unknown levels of peak CK during admission. For retrospective analysis of clinically acquired data, the Medical Ethical Committee in the Leiden University Medical Center (LUMC) waived the need for patient written informed consent. Since the data did not contain any identifiers that could be traced back to the individual patient and the data are obtained for patient care, the Dutch Central Committee on Human-Related Research permits the use of anonymous data without prior 
approval of an institutional review board. This study was conducted according to the declaration of Helsinki.

\section{Study Procedure}

The MISSION! protocol contains a standardized pre-hospital, in-hospital, and outpatient clinical framework to optimize treatment. The pre-hospital phase includes diagnosis by a high-quality 12-lead ECG. All patients were treated during the pre-hospital phase with a loading dose of aspirin $300 \mathrm{mg}$ orally and a loading dose of either clopidogrel $300 \mathrm{mg}$ orally or prasugrel $60 \mathrm{mg}$ orally. Furthermore, all patients received abciximab (dose abciximab, $0.25 \mathrm{mg} / \mathrm{kg}$ bolus followed by an infusion of $0.125 \mathrm{Ag} / \mathrm{kg}$ per minute during $12 \mathrm{~h}$ ) in the absence of contraindications. In total, $4.9 \%$ of the patients did not receive abciximab. During the in-hospital phase eligible patients were directly sent to the catheterization lab. Primary PCI was performed according to the clinical guidelines. If tolerated, within $24 \mathrm{~h}$ after admission, patients were prescribed beta-blockers, angiotensin-conversing enzyme (ACE) inhibitors, statins, acetylsalicylic acids, and thienopyridines. More than $95 \%$ of the patients received a statin, an acetylsalicylic acid, and a thienopyridine and more than $85 \%$ of the patients received a beta-blocker and an ACE inhibitor within $24 \mathrm{~h}$ after admission. For the measurement of lipid levels and cardiac biomarkers, plasma samples were obtained from included patients before pPCI was performed. Subsequently, for cardiac biomarker assessment, plasma samples with $6 \mathrm{~h}$ interval for at least $48 \mathrm{~h}$ were withdrawn. The LDL-c level was calculated from total cholesterol, triglycerides, and HDL-cholesterol concentrations, using the Friedewald formula [22]. CK activity was measured at $37^{\circ} \mathrm{C}$ with an International Federation of Clinical Chemistry (IFCC)-traceable method (Roche Diagnostics) on Modular P analyzers.

\section{Data Acquisition/Clinical Data}

Since the implementation of this protocol in February 2004, clinical and angiographic data and laboratory measurement are systematically collected for each MISSION! patient in EPDVISION, using a unique study number.

\section{Study Endpoint}

Study endpoint was defined as the enzymatic infarct size defined as the maximal level of CK measured during admission. Peak CK level was defined as the maximal CK level measured during admission, provided that maximal $\mathrm{CK}$ level is preceded and followed by lower CK levels.

\section{Statistical Analysis}

Continuous variables with a normal distribution are presented as mean \pm standard deviation. The Mann-Whitney $U$ test was used in case of two groups and the Kruskal-Wallis test was used in case of more than two groups to test differences in non-normally distributed data. These are presented as medians and interquartile range (IQR). Categorical variables are expressed as numbers and percentages. Skewed distributed outcome variables were log-transformed for linear regression analysis. At first, all variables were analyzed in a univariate linear regression model. Secondly, all variables with a $p$ value less than 0.10 in univariate analysis were included in a multivariate linear regression model. The fitted beta regression coefficients were compared with their standard errors using the $t$ test, and values and 95\% confidence intervals were calculated. All statistical tests were two-tailed, and $p$ values less than 0.05 were considered statistically significant. Analyses were conducted with SPSS 23.0 statistical analysis software (IBM, Armonk, NY, USA).

\section{RESULTS}

\section{Patient Characteristics}

In total, 2248 patients were evaluated in the current study (Fig. 1). Mean age was $61.8 \pm 12.2$ years and $562(25.0 \%)$ were female. Cardiovascular risk factors, such as current smoking (45.2\%), positive family history of 


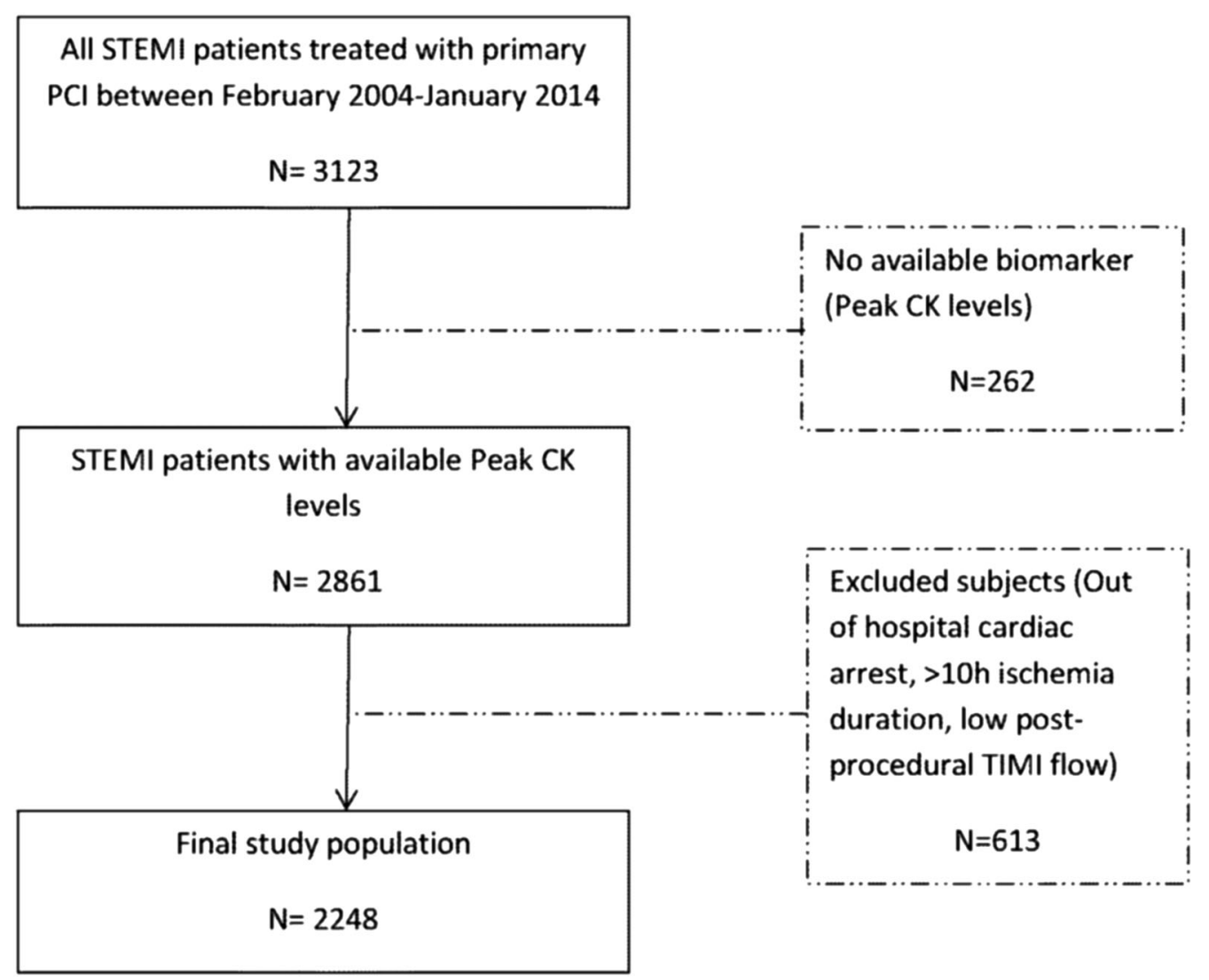

Fig. 1 Flowchart of the population. Patients were eligible if they were admitted with STEMI and treated with pPCI. Patients were excluded if peak CK level was not known, if they had an out hospital cardiac arrest (OHCA), if time of

coronary artery disease (39.7\%), and hypertension $(36.2 \%)$, were highly prevalent. Before admission $407(18.1 \%)$ patients were on statin therapy, $17.8 \%$ were on a beta-blocker, $21.0 \%$ were on an ACE inhibitor or AT2 antagonist, $1.2 \%$ were on a thienopyridine, and $16.2 \%$ were already on an acetylsalicylic acid. In total, 8.5\% had a previous myocardial infarction before admission, $6.9 \%$ had a PCI before admission, and $2.8 \%$ were known to have chronic kidney disease. All patient characteristics are demonstrated in Table 1. Data about the excluded patients $(n=875)$ are summarized in the supplementary table. Baseline and clinical characteristics of the excluded patients were, essentially, similar compared to the included patients. ischemia was at least $10 \mathrm{~h}$, or if post-procedural TIMI flow was less than 3

\section{Clinical Characteristics}

Table 2 shows the clinical characteristics of the patients. The left anterior descending (LAD) artery was identified as the culprit vessel in $42.1 \%$ of the cases; $71.2 \%$ of the patients had a TIMI flow of 0 or 1 before reperfusion in the culprit vessel. The median time of ischemia was $167 \mathrm{~min}$ (IQR $123-246 \mathrm{~min}$ ); $56.6 \%$ of the patients had multivessel disease. Mean LDL-c at baseline was $3.6 \pm 1.1 \mathrm{mmol} / \mathrm{L}$ and median peak CK was 1275 U/L (IQR 564-2590 U/L). In the group of patients using a statin before admission, the mean LDL-c was $2.74 \pm 1.0 \mathrm{mmol} / \mathrm{L}$ versus $3.80 \pm 1.0 \mathrm{mmol} / \mathrm{L}$ in the non-statin users before admission $(p<0.001)$ (results not shown). 
Table 1 Patients' characteristics

\section{Variable}

Patients characteristics

Age (years)

Female gender $n(\%)$

Cardiovascular risk factors

Current smoker, $n(\%)$

Ex-smoker, $n(\%)$

Non-insulin-dependent diabetes mellitus ${ }^{\mathrm{a}}, n(\%)$

Insulin-dependent diabetes mellitus, $n$ (\%)

Family history of coronary artery disease ${ }^{\mathrm{b}}, n(\%)$

Treated hypercholesterolemia ${ }^{\mathrm{c}}, n(\%)$

Treated hypertension $^{\mathrm{c}}, n(\%)$

Body mass index $\left(\mathrm{kg} / \mathrm{m}^{2}\right)$

Comorbidities

Previous myocardial infarction, $n$ (\%)

$192(8.5)$

Previous PCI, $n$ (\%)

$154(6.9)$

Previous CABG, $n(\%)$

$42(1.9)$

History of cerebrovascular disease, $n$ (\%)

$100(4.4)$

Chronic kidney disease ${ }^{\mathrm{d}}$

$63(2.8)$

Previous medication use

Beta-blocker, $n$ (\%)

$401(17.8)$

ACE inhibitor/AT2 antagonist, $n$ (\%)

$472(21.0)$

Statin, $n(\%)$

$407(18.1)$

Thienopyridine, $n$ (\%)

$27(1.2)$

Ascal, $n(\%)$
$365(16.2)$

Data are expressed as number (\%) or mean \pm standard deviation

a Use of glucose-lowering agents or known with glucose $>6.9 \mathrm{mmol} / \mathrm{L}$

b First-degree relative $<60$ years old with cardiovascular disease

c Explicitly stated in patient history or previous pharmacologic treatment

d Explicitly stated in patient history

\section{Correlation between Variables and Enzymatic Infarct Size}

Figure 2 shows the relation between several variables and infarct size. LDL-c values were positively associated with infarct size (Fig. 2a).
Infarct size is higher when the culprit vessel is the LAD than in patients with another vessel as culprit lesion (Fig. 2c). A low TIMI flow (0 or 1) before pPCI is significantly associated with a large infarct size (Fig. 2d). A long delay from onset symptoms to balloon time also resulted in 
Table 2 Clinical characteristics

\section{Variable}

Clinical characteristics

Left anterior descending artery as culprit artery, $n$ (\%)

Number of narrowed coronary arteries ${ }^{\mathrm{a}}, n$ (\%)

1

2

Door-to-balloon time ( $\mathrm{min})$

Median

25th, 75th percentile

Time of ischemia $(\mathrm{min})^{\mathrm{b}}$

Median

25th, 33th, 66th, 75th percentile

Killip class, $n(\%)$

1

2

3

4

Killip class $\geq 2, n(\%)$

Baseline thrombolysis in myocardial infarction flow, $n(\%)^{\mathrm{c}}$

0

1

2

3

Baseline thrombolysis in myocardial infarction flow $<2$, $n(\%)$

Laboratory results

Peak creatine kinase $(\mathrm{U} / \mathrm{L})$

Median

25th, 75th percentile

Peak cardiac troponin $\mathrm{T}(\mu \mathrm{g} / \mathrm{L})$

Median

25th, 75th percentile

Estimated glomerular filtration rate $\left(\mathrm{ml} / \mathrm{min} / 1.73 \mathrm{~m}^{2}\right)$

Estimated glomerular filtration rate $\leq 60\left(\mathrm{ml} / \mathrm{min} / 1.73 \mathrm{~m}^{2}\right), n(\%)$

LDL-cholesterol $(\mathrm{mmol} / \mathrm{L})$
1275

3.39

All patients $(n=2248)$

$947(42.1)$

$968(43.1)$

798 (35.5)

46

34, 68

167

123, 136, 210, 246

$2119(94.3)$

60 (2.7)

$11(0.5)$

$25(1.1)$

$96(4.3)$

$1312(58.4)$

$287(12.8)$

$327(14.6)$

311 (13.8)

$1599(71.1)$

564, 2590

$1.30,7.15$

$75.0 \pm 22.6$

149 (6.6)

$3.6 \pm 1.1$ 
Table 2 continued

\begin{tabular}{lc}
\hline Variable & All patients $(\boldsymbol{n}=\mathbf{2 2 4 8})$ \\
\hline HDL-cholesterol (mmol/L) & $1.2 \pm 0.4$ \\
Triglycerides (mmol/L) & $1.9 \pm 1.3$ \\
Total cholesterol (mmol/L) & $5.3 \pm 1.2$ \\
\hline
\end{tabular}

Data are expressed as number (\%), mean \pm standard deviation, or median (interquartile range)

a A narrowed coronary artery was defined as a stenosis of $\geq 50 \%$ on baseline coronary angiogram

b Symptom onset to time of reperfusion of the culprit lesion during PCI (in minutes)

c Baseline thrombolysis in myocardial infarction (TIMI) flow is the TIMI flow before primary PCI in the culprit vessel
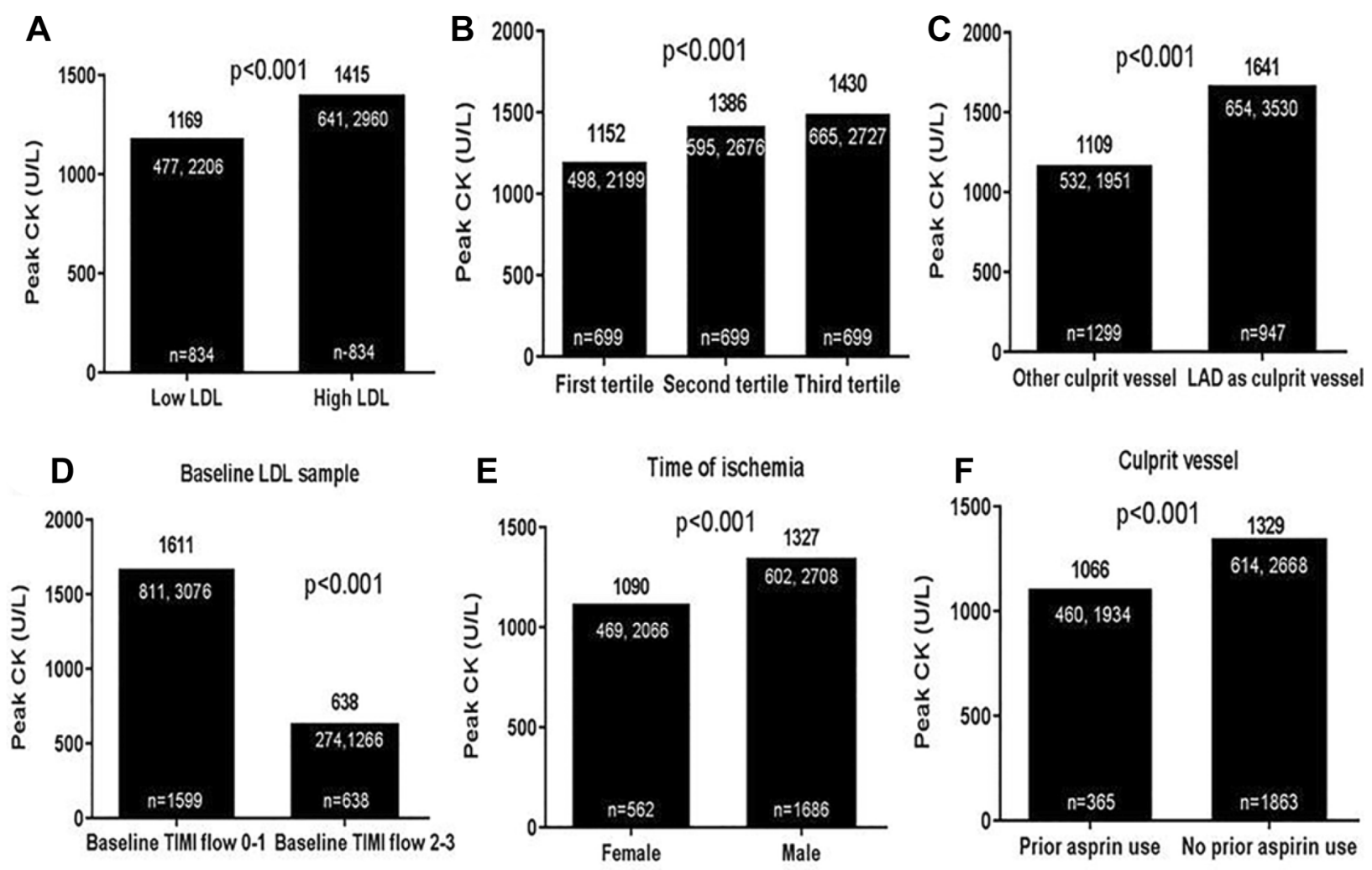

Baseline TIMI flow

Gender

Use of aspirin

Fig. 2 Impact of variable on infarct size. Peak CK expressed as median with interquartile range. a Infarct size in patients having LDL-cholesterol below median $(3.61 \mathrm{mmol} / \mathrm{L})$ and above the median. b Total symptom onset to balloon time divided into three tertiles, first tertile $<138 \mathrm{~min}$, second tertile $138-215 \mathrm{~min}$, third tertile

a large infarct size (Fig. 2b). Median infarct size in male patients was larger than that in female patients (Fig. 2e) and prior aspirin use was associated with smaller infarct size (Fig. 2f).
216-600 min. c Influence of culprit artery on infarct size. d Influence of baseline TIMI flow on infarct size. e Influence of gender on infarct size. $\mathbf{f}$ Influence of the use of aspirin prior to myocardial infarction on infarct size. LAD, left anterior descending artery; Baseline TIMI flow, TIMI flow before primary PCI in the culprit vessel

Table 3 shows the univariate and multivariate variables that correlate with peak CK level. Multivariate analysis showed that LDL-c levels were independently associated with peak CK 
Table 3 Independent correlates for infarct size measured by (log transformed) peak CK

\begin{tabular}{|c|c|c|c|c|c|c|}
\hline \multirow[t]{2}{*}{ Variable } & \multicolumn{3}{|c|}{ Univariate analysis } & \multicolumn{3}{|c|}{ Multivariate analysis } \\
\hline & $\bar{\beta}$ & 95\% CI & $P$ & $\bar{\beta}$ & $95 \% \mathrm{CI}$ & $P$ \\
\hline Age & -0.003 & -0.004 to 0.001 & 0.001 & -0.001 & -0.003 to 0.001 & 0.315 \\
\hline Gender (male) & 0.091 & 0.045 to 0.136 & $<0.001$ & 0.103 & 0.052 to 0.154 & $<0.001$ \\
\hline Current smoker & 0.050 & 0.011 to 0.090 & 0.013 & 0.036 & -0.009 to 0.082 & 0.119 \\
\hline Ex-smoker & -0.048 & -0.108 to 0.013 & 0.121 & & & \\
\hline Diabetes mellitus & -0.043 & -0.105 to 0.018 & 0.168 & & & \\
\hline Previous myocardial infarction & -0.039 & -0.109 to 0.031 & 0.278 & & & \\
\hline Prior statin therapy & -0.087 & -0.138 to -0.035 & 0.001 & -0.003 & -0.072 to 0.065 & 0.923 \\
\hline Prior aspirin therapy & -0.098 & -0.151 to -0.044 & $<0.001$ & -0.073 & -0.146 to 0.000 & 0.050 \\
\hline Prior beta-blocker therapy & -0.073 & -0.124 to -0.022 & 0.005 & -0.012 & -0.075 to 0.051 & 0.705 \\
\hline LAD as culprit artery & 0.140 & 0.100 to 0.179 & $<0.001$ & 0.152 & 0.108 to 0.195 & $<0.001$ \\
\hline Three vessel disease & -0.001 & -0.049 to 0.042 & 0.981 & & & \\
\hline Time of ischemia $^{a}$ & 0.177 & 0.080 to 0.274 & $<0.001$ & 0.206 & 0.102 to 0.309 & $<0.001$ \\
\hline Baseline TIMI flow 0-1 & 0.394 & 0.354 to 0.435 & $<0.001$ & 0.431 & 0.384 to 0.478 & $<0.001$ \\
\hline LDL-cholesterol $(\mathrm{mmol} / \mathrm{L})$ & 0.060 & 0.039 to 0.081 & $<0.001$ & 0.041 & 0.019 to 0.062 & $<0.001$ \\
\hline
\end{tabular}

TIMI thrombolysis in myocardial infarction flow, LAD Left anterior descending, $\beta$ standardized regression coefficient

a Symptom onset to balloon time (in minutes)

level $\quad[\beta=0.041 \quad(95 \% \quad$ CI $\quad 0.019-0.062)$, $p<0.001]$. In addition, male gender $[\beta=0.103$ (95\% CI 0.052-0.154), $p<0.001]$, culprit lesion in $\mathrm{LAD}[\beta=0.152 \quad(95 \% \quad \mathrm{CI} \quad 0.108-0.195)$, $p<0.001]$, ischemic time $[\beta=0.206(95 \% \mathrm{CI}$ $0.102-0.309), p=0.001]$, and baseline TIMI flow $0-1[\beta=0.431$ (95\% CI 0.384-0.478), $p<0.001]$ were independently associated with peak CK level. Prior aspirin therapy was significantly associated with lower peak CK level $[\beta=-0.073 \quad(95 \% \quad$ CI -0.146 to 0.000$)$, $p=0.050]$. Statin use before admission was in univariate analysis associated with a lower infarct size; however, this effect perished in multivariate analysis.

\section{DISCUSSION}

The purpose of this large cohort study was to assess whether infarct size in patients with STEMI treated with pPCI is determined by pre- existing factors. The primary finding of this study is that higher levels of LDL-c at the time of admission are independently associated with greater infarct size expressed as peak CK level. In addition, anterior infarction, time of ischemia, low pre-procedural TIMI flow, gender, and previous use of aspirin are factors that are related to infarct size in STEMI patients after pPCI.

Further understanding of the variables that affect infarct size in STEMI patients after pPCI can have important implications for patients' treatment and prognosis. Owing to improvements of diagnosis, therapy, and care, mortality rates of STEMI patients are reduced at the expense of increasing numbers of STEMI patients with heart failure. This makes it essential to understand what factors are associated with infarct size, particularly if these factors are potentially modifiable, as this could lead to the earlier detection and development of advanced therapies. 
This study demonstrates that higher LDL-c levels are associated with greater infarct size in STEMI patients treated with pPCI. Besides high LDL-c levels, anterior infarction, time of ischemia, low pre-procedural TIMI flow, and male gender are shown to be associated with larger infarct size. These determinants are well-established risk factors for a larger infarct size which were earlier identified in a pooled analysis of four randomized STEMI trials by Stone et al. [2]. Since the mechanisms underlying the effect of higher LDL-c levels on infarct size are still unclear, some explanations may be suggested. Our hypothesis is that a higher LDL-c level itself may aggravate MRI. Several studies with animal models reported that hypercholesterolemia could aggravate ischemia/reperfusion injury [9-12, 23], leading to a greater infarct size. Several mechanisms underlying the relation between hypercholesterolemia and increased myocardial reperfusion injury have been proposed; for example, by increased oxidative stress $[10,23]$, reduced extent of the cardioprotective effect of HDL-c [9], activated endoplasmic reticulum stress-mediated apoptosis [12], or by upregulation of inflammatory processes [24]. In human patients, exploration of the link between hypercholesterolemia and infarct size is limited [25-27] and moreover these studies were not able to consistently confirm the results from animal studies. Marenzi et al. conducted a prospective cohort study which evaluated the effect of statin therapy on myocardial infarct size assessed with cardiac magnetic resonance (CMR) in patients treated with pPCI for STEMI [25]. They observed no significant association between infarct size and LDL-c levels at hospital admission. Some studies suggest that ischemic preconditioning is dependent of serum LDL-C levels present at the time of reperfusion $[26,27]$. Ischemic preconditioning is defined by initiating periods of transient myocardial ischemia and reperfusion before the sustained ischemic episode [27]. Ischemic preconditioning is a form of cardioprotection, and appears to inhibit lethal reperfusion injury $[26,27]$. Kyriakides et al. [26] tested the hypothesis that hyperlipidemia inhibits the reduction of myocardial ischemia normally observed after repeated balloon inflations during angioplasty. They showed that hyperlipidemia prevents the reduction of myocardial ischemia on repeated balloon inflations during angioplasty. A similar study, performed by Ungi et al. [27], scrutinized the effect of high serum cholesterol levels on ischemic preconditioning by means of beat-tobeat analysis of ST segments and found that hyperlipidemia accelerates the evolution of myocardial ischemia and delays recovery upon reperfusion.

Aspirin use prior to a MI was associated with smaller infarct size. To our knowledge no earlier studies were able to show this association. Marenzi et al., for example, found no association between prior aspirin use and infarct size determined with CMR [25]. Their relatively small population may be the reason for the lack association. Furthermore, in the present study most of the patients with an earlier MI were on aspirin therapy, which might explain why the infarct size was smaller in the patients on aspirin therapy. As a result of earlier damage to the myocardium, CK release might be less than in the case of first STEMI.

As it is generally believed that a substantial part of the myocardial infarct size is determined by MRI [17], several studies were initiated with the aim to identify cardioprotective agents that may reduce irreversible cell damage upon reperfusion $[17,28]$. As a result of its pleiotropic effects [29], statin treatment prior to primary PCI in MI patients was proposed as a potential agent that could have an effect on reperfusioninduced cell damage; however, this effect is still controversial as they yielded conflicting results. Several hypotheses could explain the potential effect of statin pre-treatment on reperfusioninduced cell damage and consequently on clinical outcome. For example, statins are known to suppress active plaque inflammation $[30,31]$, inhibit thrombosis [32], improve endothelial function [33], inhibit cell adhesion [34], and improve microvascular function $[35,36]$, all of which contribute to improved clinical outcome after PCI [37].

In the present study, statin pre-treatment was associated with lower peak CK level in univariate analysis, but vanished after multivariate analysis. Several animal studies demonstrated a beneficial effect of statin therapy on 
infarct size after reperfusion [28, 38]; yet, these results could not consistently be reproduced in patients with STEMI $[25,37,39,40]$. These conflicting results could be explained by several reasons. First, the right timing of the statin administration prior to STEMI was diverse, but is of importance since it takes $2-5 \mathrm{~h}$ for statins to reach an optimal blood level during reperfusion [41, 42]. Secondly, the intensity of the statin therapy necessary to attenuate the infarct size differed between the studies. An earlier study conducted in pigs showed that pre-treatment with $160 \mathrm{mg}$ of oral rosuvastatin reduced infarct size, whereas $80 \mathrm{mg}$ had no significant effect [43]. Finally, in STEMI patients' myocardial ischemic injury might be too severe to be prevented or reduced [37].

On the other hand, LDL-c level was significantly lower in patients treated with statin before admission than in patients without statin therapy, which may indicate that not statin use but LDL-c level itself is related to infarct size. Further research, with well-conducted randomized trials that address the issues of timing and the intensity of the statin therapy, is needed in STEMI patients to explore the full potential of pre-treatment with statin therapy and other potential cardioprotective agents that can reduce irreversible cell damage upon reperfusion.

As expected, a high incidence of traditional risk factors such as smoking, hypertension, and dyslipidemia was present in this cohort. Elevated LDL-c is an important risk factor for the development of atherosclerosis and subsequent ischemic heart disease [7], and the association between these risk factors and cardiovascular mortality has been known for decades [44]. Therefore, identifying high-risk patients before they develop ischemic heart disease is crucial. The results of this study emphasize not only that primary prevention and timely management of patients with high cardiovascular risk profiles have beneficial effects on the development of ischemic heart disease but also that early identification of patients with high LDL-c levels combined with lifestyle changes and optimal medical treatment might lead to an improved prognosis after having developed ischemic heart disease.
Several limitations of the present study should be mentioned. First, since an observational cohort study was conducted, we could not account for undocumented clinical variables which may possibly have influenced the outcomes. Although this study is retrospectively conducted, all patients are treated according to the institutional MISSION! which provides an integrated approach of MI care to optimize treatment. This yielded in a very homogeneous STEMI population which resulted in a very high number of patients being treated according to the guidelines. Secondly, patients with known coronary artery disease or patients with a previous MI were not excluded in our analysis. In this manner, we believed that it reflects a population-based cohort in the best possible way. Thirdly, information about the number of patients either receiving prasugrel of clopidogrel was not available for this study. Theoretically, prasugrel, a more potent antiplatelet drug, could lead to a smaller infarct size. Furthermore, information about statin compliance, intensity of the statin treatment, and treatment duration prior to MI was not available for this study. This could explain the confined association between statin pre-treatment and lower peak CK levels in this study.

Finally, peak CK levels during admission were used as an estimate of infarct size. Peak serum CK levels can be used to estimate infarct size if reperfusion is established rapidly and successfully [14-16] and peak CK is, compared to cardiac magnetic resonance imaging (cMRI), more easily implemented in daily practice and far less expensive. However, cMRI has emerged as a well-established technique for quantifying myocardial infarct size, has been shown to correlate well with clinical outcome, and is the gold standard. Therefore, further studies are warranted to establish the association between cholesterol levels and infarct size on cMRI.

\section{CONCLUSION}

This study demonstrates that besides the more established predictors of infarct size, elevated LDL-c is associated with augmented infarct size in patients with STEMI. Other techniques, such 
as cMRI, should strengthen the evidence that pre-existing LDL-c levels influence infarct size. Furthermore, in larger patient cohorts it should be established whether elevated pre-infarct LDLc levels lead to worse clinical outcome and how their prognosis after having developed MI can be improved.

\section{ACKNOWLEDGEMENTS}

We thank the participants of the study.

Funding. No funding or sponsorship was received for this study or publication of this article.

Authorship. All named authors meet the International Committee of Medical Journal Editors (ICMJE) criteria for authorship for this article, take responsibility for the integrity of the work as a whole, and have given their approval for this version to be published.

Disclosures. Mathijs C. Bodde, Maaike P. J. Hermans, Ron Wolterbeek, Christa M. Cobbaert, Arnaud van der Laarse and Martin J. Schalij have nothing to disclose. J. Wouter Jukema is a member of the journal's Editorial Board.

Compliance with Ethics Guidelines. For retrospective analysis of clinically acquired data, the Medical Ethical Committee in the Leiden University Medical Center (LUMC) waived the need for patient written informed consent. Since the data did not contain any identifiers that could be traced back to the individual patient and the data are obtained for patient care, the Dutch Central Committee on Human-Related Research permits the use of anonymous data without prior approval of an institutional review board. This study was conducted according to the declaration of Helsinki.

Data Availability. The datasets generated during and/or analyzed during the current study are not publicly available due to bad experiences regarding data hustling and cherry picking, but request for collaborations are certainly welcome.

Open Access. This article is distributed under the terms of the Creative Commons Attribution-NonCommercial 4.0 International License (http://creativecommons.org/licenses/ by-nc/4.0/), which permits any noncommercial use, distribution, and reproduction in any medium, provided you give appropriate credit to the original author(s) and the source, provide a link to the Creative Commons license, and indicate if changes were made.

\section{REFERENCES}

1. Keeley EC, Boura JA, Grines CL. Primary angioplasty versus intravenous thrombolytic therapy for acute myocardial infarction: a quantitative review of 23 randomised trials. Lancet. 2003;361(9351):13-20.

2. Stone GW, Dixon SR, Grines CL, et al. Predictors of infarct size after primary coronary angioplasty in acute myocardial infarction from pooled analysis from four contemporary trials. Am J Cardiol. 2007;100(9):1370-5.

3. Sakakura K, Nakano M, Otsuka F, Ladich E, Kolodgie FD, Virmani R. Pathophysiology of atherosclerosis plaque progression. Heart Lung Circ. 2013;22(6):399-411.

4. Libby P. Inflammation in atherosclerosis. Nature. 2002;420(6917):868-74.

5. Scandinavian Simvastatin Survival Study Group. Randomised trial of cholesterol lowering in 4444 patients with coronary heart disease: the Scandinavian Simvastatin Survival Study (4S). Lancet. 1994;344(8934):1383-9.

6. Ridker PM, Danielson E, Fonseca FA, et al. Rosuvastatin to prevent vascular events in men and women with elevated C-reactive protein. New Engl J Med. 2008;359(21):2195-207.

7. Anderson KM, Castelli WP, Levy D. Cholesterol and mortality. 30 years of follow-up from the Framingham study. JAMA. 1987;257(16):2176-80.

8. Shepherd J, Cobbe SM, Ford I, et al. Prevention of coronary heart disease with pravastatin in men with hypercholesterolemia. West of Scotland Coronary Prevention Study Group. New Engl J Med. 1995;333(20):1301-7. 
9. Vilahur G, Gutierrez M, Casani L, et al. Hypercholesterolemia abolishes high-density lipoproteinrelated cardioprotective effects in the setting of myocardial infarction. J Am Coll Cardiol. 2015;66(21):2469-70.

10. Osipov RM, Bianchi C, Feng J, et al. Effect of hypercholesterolemia on myocardial necrosis and apoptosis in the setting of ischemia-reperfusion. Circulation. 2009;120(11 Suppl):S22-30.

11. Golino P, Maroko PR, Carew TE. The effect of acute hypercholesterolemia on myocardial infarct size and the no-reflow phenomenon during coronary occlusion-reperfusion. Circulation. 1987;75(1):292-8.

12. Wu N, Zhang X, Jia P, Jia D. Hypercholesterolemia aggravates myocardial ischemia reperfusion injury via activating endoplasmic reticulum stress-mediated apoptosis. Exp Mol Pathol. 2015;99(3):449-54.

13. Tzivoni D, Koukoui D, Guetta V, Novack L, Cowing G. Comparison of troponin T to creatine kinase and to radionuclide cardiac imaging infarct size in patients with ST-elevation myocardial infarction undergoing primary angioplasty. Am J Cardiol. 2008;101(6):753-7.

14. Dohi T, Maehara A, Brener SJ, et al. Utility of peak creatine kinase-MB measurements in predicting myocardial infarct size, left ventricular dysfunction, and outcome after first anterior wall acute myocardial infarction (from the INFUSE-AMI trial). Am J Cardiol. 2015;115(5):563-70.

15. Chia S, Senatore F, Raffel OC, Lee H, Wackers FJ, Jang IK. Utility of cardiac biomarkers in predicting infarct size, left ventricular function, and clinical outcome after primary percutaneous coronary intervention for ST-segment elevation myocardial infarction. JACC Cardiovasc Interv. 2008;1(4):415-23.

16. Nienhuis MB, Ottervanger JP, de Boer MJ, et al. Prognostic importance of creatine kinase and creatine kinase-MB after primary percutaneous coronary intervention for ST-elevation myocardial infarction. Am Heart J. 2008;155(4):673-9.

17. Yellon DM, Hausenloy DJ. Myocardial reperfusion injury. New Engl J Med. 2007;357(11):1121-35.

18. Liem SS, van der Hoeven BL, Oemrawsingh PV, et al. MISSION!: optimization of acute and chronic care for patients with acute myocardial infarction. Am Heart J. 2007;153(1):14.e1-2.

19. O'Gara PT, Kushner FG, Ascheim DD, et al. 2013 ACCF/AHA guideline for the management of STelevation myocardial infarction: a report of the American College of Cardiology Foundation/
American Heart Association Task Force on Practice Guidelines. J Am Coll Cardiol. 2013;61(4):e78-140.

20. Steg PG, James SK, Atar D, et al. ESC guidelines for the management of acute myocardial infarction in patients presenting with ST-segment elevation. Eur Heart J. 2012;33(20):2569-619.

21. Thygesen K, Alpert JS, Jaffe AS, et al. Third universal definition of myocardial infarction. J Am Coll Cardiol. 2012;60(16):1581-98.

22. DeLong DM, DeLong ER, Wood PD, Lippel K, Rifkind BM. A comparison of methods for the estimation of plasma low- and very low-density lipoprotein cholesterol. The Lipid Research Clinics Prevalence Study. JAMA. 1986;256(17):2372-7.

23. Prasad K, Kalra J. Experimental atherosclerosis and oxygen free radicals. Angiology. 1989;40(9):835-43.

24. Liu HR, Tao L, Gao E, et al. Rosiglitazone inhibits hypercholesterolaemia-induced myeloperoxidase upregulation-a novel mechanism for the cardioprotective effects of PPAR agonists. Cardiovasc Res. 2009;81(2):344-52.

25. Marenzi G, Cosentino N, Cortinovis $S$, et al. Myocardial infarct size in patients on long-term statin therapy undergoing primary percutaneous coronary intervention for ST-elevation myocardial infarction. Am J Cardiol. 2015;116(12):1791-7.

26. Kyriakides ZS, Psychari S, Iliodromitis EK, Kolettis TM, Sbarouni E, Kremastinos DT. Hyperlipidemia prevents the expected reduction of myocardial ischemia on repeated balloon inflations during angioplasty. Chest. 2002;121(4):1211-5.

27. Ungi I, Ungi T, Ruzsa Z, et al. Hypercholesterolemia attenuates the anti-ischemic effect of preconditioning during coronary angioplasty. Chest. 2005;128(3):1623-8.

28. Eefting F, Rensing B, Wigman J, et al. Role of apoptosis in reperfusion injury. Cardiovasc Res. 2004;61(3):414-26.

29. Takemoto M, Liao JK. Pleiotropic effects of 3-hydroxy-3-methylglutaryl coenzyme a reductase inhibitors. Arterioscler Thromb Vasc Biol. 2001;21(11):1712-9.

30. Albert MA, Danielson E, Rifai N, Ridker PM. Effect of statin therapy on C-reactive protein levels: the pravastatin inflammation/CRP evaluation (PRINCE): a randomized trial and cohort study. JAMA. 2001;286(1):64-70.

31. McTaggart F. Comparative pharmacology of rosuvastatin. Atheroscler Suppl. 2003;4(1):9-14. 
32. Undas A, Brummel KE, Musial J, Mann KG, Szczeklik A. Simvastatin depresses blood clotting by inhibiting activation of prothrombin, factor $\mathrm{V}$, and factor XIII and by enhancing factor Va inactivation. Circulation. 2001;103(18):2248-53.

33. Dimmeler S, Aicher A, Vasa M, et al. HMG-CoA reductase inhibitors (statins) increase endothelial progenitor cells via the PI 3-kinase/Akt pathway. J Clin Investig. 2001;108(3):391-7.

34. Patti G, Chello M, Pasceri V, et al. Protection from procedural myocardial injury by atorvastatin is associated with lower levels of adhesion molecules after percutaneous coronary intervention: results from the ARMYDA-CAMs (Atorvastatin for Reduction of MYocardial Damage during AngioplastyCell Adhesion Molecules) substudy. J Am Coll Cardiol. 2006;48(8):1560-6.

35. Kim JS, Kim J, Choi D, et al. Efficacy of high-dose atorvastatin loading before primary percutaneous coronary intervention in ST-segment elevation myocardial infarction: the STATIN STEMI trial. JACC Cardiovasc Interv. 2010;3(3):332-9.

36. Paraskevaidis IA, Iliodromitis EK, Ikonomidis I, et al. The effect of acute administration of statins on coronary microcirculation during the prerevascularization period in patients with myocardial infraction. Atherosclerosis. 2012;223(1):184-9.

37. Ko YG, Won H, Shin DH, et al. Efficacy of early intensive rosuvastatin therapy in patients with STsegment elevation myocardial infarction undergoing primary percutaneous coronary intervention (ROSEMARY study). Am J Cardiol. 2014;114(1):29-35.
38. Bell RM, Yellon DM. Atorvastatin, administered at the onset of reperfusion, and independent of lipid lowering, protects the myocardium by up-regulating a pro-survival pathway. J Am Coll Cardiol. 2003;41(3):508-15.

39. Kim JW, Yun KH, Kim EK, et al. Effect of high dose rosuvastatin loading before primary percutaneous coronary intervention on infarct size in patients with ST-segment elevation myocardial infarction. Korean Circ J. 2014;44(2):76-81.

40. Post S, Post MC, van den Branden BJ, et al. Early statin treatment prior to primary PCI for acute myocardial infarction: REPERATOR, a randomized placebo-controlled pilot trial. Catheter Cardiovasc Interv. 2012;80(5):756-65.

41. Bellosta S, Paoletti R, Corsini A. Safety of statins: focus on clinical pharmacokinetics and drug interactions. Circulation. 2004;109(23 Suppl 1):50-7.

42. Martin PD, Mitchell PD, Schneck DW. Pharmacodynamic effects and pharmacokinetics of a new HMG-CoA reductase inhibitor, rosuvastatin, after morning or evening administration in healthy volunteers. Br J Clin Pharmacol. 2002;54(5):472-7.

43. Bulhak AA, Gourine AV, Gonon AT, Sjoquist PO, Valen G, Pernow J. Oral pre-treatment with rosuvastatin protects porcine myocardium from ischaemia/reperfusion injury via a mechanism related to nitric oxide but not to serum cholesterol level. Acta Physiol Scand. 2005;183(2):151-9.

44. Shekelle RB, Shryock AM, Paul O, et al. Diet, serum cholesterol, and death from coronary heart disease. The Western Electric study. New Engl J Med. 1981;304(2):65-70. 